\title{
The Study of Personalized Intelligent Cooperative Teaching Service System Based on Grid
}

\author{
San-jun Liu ${ }^{a}$,Yan-feng Zhang ${ }^{b}$ \\ Institute of Information Engineering of Jiaozuo University; Jiaozuo, Henan province,China \\ aliusanjun1975@163.com, bchinazhyf@163.com
}

Key words: PSPTS; Cooperative Teaching Service; grid; personalized service

\begin{abstract}
By studying the theory and method of the Grid Services and analyzing the drawbacks of the traditional teaching system, the article presents a personalized intelligent cooperative teaching service system based on grid. Simultaneously, it makes use of the grid technology to detailed design the teaching service engine. The engine is the core component of the system, it break down and mix of the distributed teaching service sources to provide the advantage of free the usual limitation of space and regions and can work at any time and at any place. The prototype PSPTS software system is developed which can provide useful reference and experiences for institutions or the relevant researches of the enterprise professional training system.
\end{abstract}

\section{Introduction}

With the rapid development of information technology and the rapid worldwide spread of the Internet technology, and the continuous change of the social talent needs, the traditional mode of teaching and education, and the information sharing mode of teaching resources can not meet the needs of the rapid social development. The current information sharing mode of teaching resources is basically to store the resources in HTML format in the network, and the storage mode of a variety of resources in different enviorments heavily relies on the operating system, platform constraints and other limitation that can not be sharded within a larger range, and can not be truly intelligently integrated. Because of these problems, the resources such as teachers, teaching contents,ideas and so on are waste seriously. Therefore, we urgently need an information sharing mode for integrated, intelligent teaching service mode to achieve an intelligent integration of the service and teaching resources, and provide a good foundation for dynamic virtual learning organization. 


\section{Design of personalized intelligent cooperative teaching service system based on the grid technology}

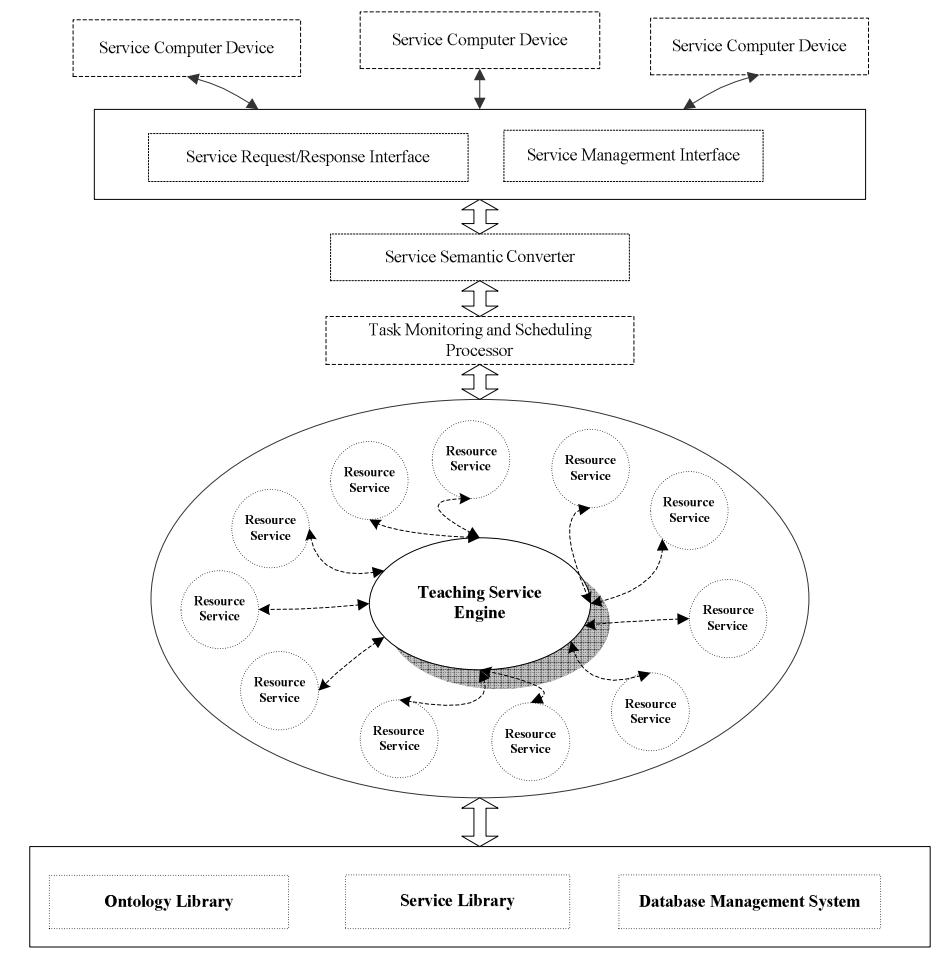

Fig 1 PSPTS system framework based on the grid technology

The personalized intelligent cooperative teaching service system (PSPTS) based on the grid is a virtual teaching and service system which is built with a combination of the grid technology in a normal computing enviroment. The main system architecture of the system is shown as Fig 1, and the main design purpose is to gather the distributed teaching resources and achieve the sharing of the teaching information resource to form a personalized intelligent teaching service.

The various of teaching and service resources of the grid cooperatively work under the control of the teaching and service engine. As a grid node in the teaching resources network, the basic structure of the grid on the node consists of three parts: the local teaching resource services, grid servers and grid application systems. As a basic structure of the local grid service, the local teaching resource servers can be heterogeneous, and must comply with the operation environment of the grid.

The most basic function achived in the teaching service resource grid is modular services, which specifically comprise the following main points:

data query service. There are two kinds of query services are provided for the students and the resource users, and one is the inquiry of teaching resources for teachers, whose main function is to provide back-up resources for the teaching of the teachers, and provide a basis for the formation of the whole curriculum system. The other is the inquiry of curriculum for students, the system analyzes the teaching resource services according to the needs of the personalized study of the students, and searches the teaching service resources in the grid according to the analyzing result, and re-conbines the resources to form the teaching curriculum system to facilitate the students to study.

analysis of teaching resources. The System does different service requirement analysis according to different user roles. On one hand, the servie analysis for the students is based on the formal description for the learning task, a various of teaching reource services can be decomposed and combined on the basis of the decomposition of the learning unit task to form personalized learning mode and generate the sequence of events, which can provide teaching service activities for the students at anytime and in anywhere without the space and geographical constraints. The teaching activities mainly include video lessons, study notes, test questions, etc.. On the other 
hand, the resource service analysis is mainly based on the formal description for the teaching service task, and the teaching service resources in the grid are re-combined by decomposing the teaching unit task, to form a curriculum lesson system which meet the teaching requirement of the teachers. In this process, the teachers do not need to worry about the lack of the teaching resources, and can integrate and collaboratively use the teaching resources distributed in different geographical locations.

\section{The assignment model of the system roles}

Considering the allocation and use of the resources, the user roles can be divided into three kinds of managers, resource providers and resource users.

The core of the user rolesis the managers of the system resources and the teaching services. The managers of the system resources is responsible for maintaining resources submitted by all users, and the providers of the teaching resources mainly are teachers. The teaching materials can be submitted by a variety of computing devices (mobile phones, Pocket PC, PDA, computer, etc.) ater sucessfully logining. These materials such as lesson plans, electronic courseware, video lesson plans are registered in the teaching resources according to uniform service model. The users of the teaching resources also are the users of the teaching services, and the users can do daily teaching activities such as querying the lesson plans, searching the resources, online teaching, generating test database. The users of the teaching resources can learn online at anytime and in anywhere, and discuss online, and other communication activities. When the teachers travel abroad, they can online answer the questions submitted by the students by using the PDA, mobile phones and other mobile devices. The students can also communicate and discuss by mobile phones.

\section{Design and principles analysis of the teaching service engine}

The teaching service engine is the core of th ewhole system, its structure is shown in Fig 2, and the design is primarily a multi-layer archiecture.

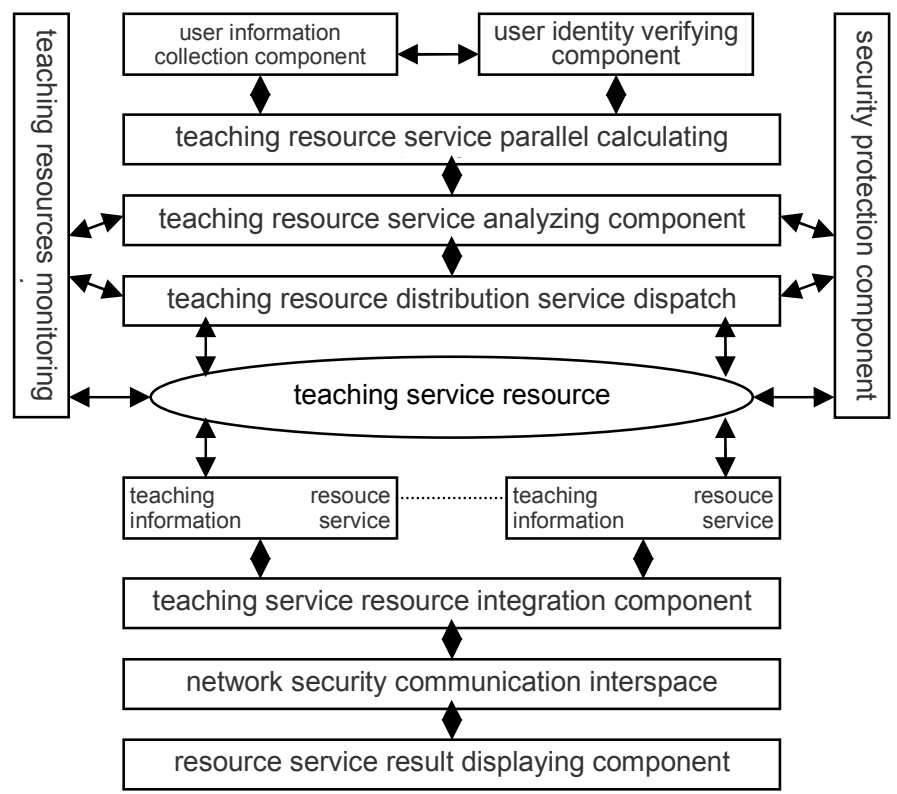

Fig 2 Cooperative teaching service system

This architecture gathers the computing resources distributed in different grids of the teaching service and teaching resources, and maximum provides a virtual computing environment for the users and applications. From the functional point, this architecutre can be divided into ten functional components, when a user logins the system, the relevant authentication information is submitted, and the user identity is verified by the user information collection component. If the user identity is 
legal, the requirement informattion submitted by the user will be analyzed by the teaching service analysis component via the interface of teaching resources and services. The analysis result is delivered to the service dispatch component by the teaching service anaolysis component to search resources, then the resources are dispatched according to the different needs of the users by the distribution service dispatch component based on the result analyzed by the teaching resource analysis component, and the adjacent appropriate resources are chosen to be integrated according to the relevant dispatch algorithm, and finally, the resources service result is formed and is displayed by the resources service result display component. Throughout the entire operation process of the engine, there are two important components, ie, the security protection component and teaching resource monitioring component which play a very important role. The security protection component is used to protect the information and systems security of the system, prevent the information leakage, viruses and other malicious attacks. The teaching resource monitioring component is used to constantly monitior the changes and updates of the user needs, respond to the user needs, and timely reflect the changes of the information to the relevant components to deal.

\section{Conclusion}

This article provides a personalized intelligent cooperative teaching service system based on grid, on the basis of analyzing the shortcomings of the traditional teaching service system, and does a detailed design for the teaching service engine. The teaching service engine analyzes the teaching service of the users based on the formal description of the teaching tasks and the learning tasks, decomposes and combines various distributed teaching service resources based on decomposing the teaching and learning tasks to form a personalized learning model and generate the personalized intelligent cooperative teaching service system of the sequence of events. The system has no space and geographical constraints, and can do the teaching activities at anytime and in anywhere. Furthermore, the system can integrate the teaching resource in different geographical locations together to cooperatively use or publish without the worry of the lack of the teaching resources. The service framework can generate the cooperative teaching service system based on the various of teaching resources integrated in the grid, and the system is not only consistent with the cognitive level of the group of learners, but also consistent with the personality characteristics of the individual learners. The system framework will have a very good learning and reference affect for the research and development staffs of the training systems of the relevant institutions and enterprises.

\section{References}

[1] Foster I, Kesselman C, The Grid: Blueprint for a New Computing Infrastructure (2nd ed.) Beijing: Elsevier Inc, China Machine Press, 2004.

[2] Du Zhi-hui, Chen Yu, Liu Peng. Grid [M]. Beijing: Tsinghua University Press,2002:3-16

[3] Lijun Mei, Chan, WK, Tse, TH A tale of clouds: paradigm comparisons and some thoughts on research issues [C]. 2008 IEEE Asia-Pacific Services Computing Conference (APSCC 2008)

[4] Lin Li-yu, Chen Yun-hai, Zhang Min, etc. Cloud computing and operational feasibility analysis [J]. Communication hot spots, 2008, (12) 